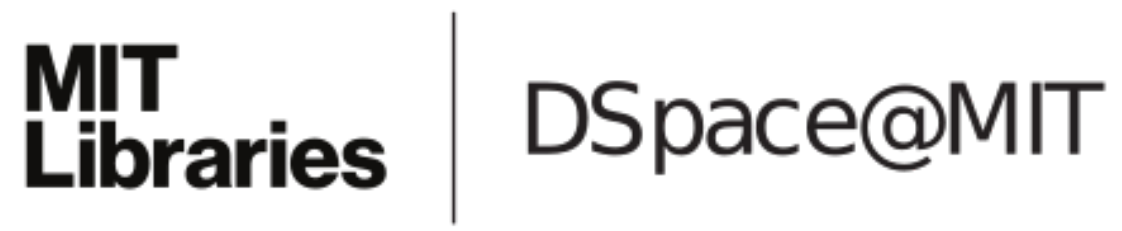

\author{
MIT Open Access Articles
}

A hidden semi-Markov model for estimating burst suppression EEG

The MIT Faculty has made this article openly available. Please share how this access benefits you. Your story matters.

Citation: Chakravarty, Sourish et al. "A hidden semi-Markov model for estimating burst suppression EEG." 41st Annual International Conference of the IEEE Engineering in Medicine and Biology Society (EMBC), July 2019, Berlin, Germany, Institute of Electrical and Electronics Engineers (IEEE), October 2019 @ 2019 IEEE

As Published: http://dx.doi.org/10.1109/embc.2019.8856802

Publisher: Institute of Electrical and Electronics Engineers (IEEE)

Persistent URL: https://hdl.handle.net/1721.1/123326

Version: Author's final manuscript: final author's manuscript post peer review, without publisher's formatting or copy editing

Terms of use: Creative Commons Attribution-Noncommercial-Share Alike 


\title{
A hidden semi-Markov model for estimating burst suppression EEG
}

\author{
Sourish Chakravarty ${ }^{a, 1}$, Taylor E. Baum ${ }^{a, 2}$, Jingzhi $\mathrm{An}^{4,7}$, Pegah Kahali ${ }^{1,5}$, \\ Emery N. Brown ${ }^{1,3,5-7}$
}

\begin{abstract}
Burst suppression is an electroencephalogram (EEG) pattern associated with profoundly inactivated brain states characterized by cerebral metabolic depression. This pattern is distinguished by short-duration band-limited electrical activity (bursts) interspersed between relatively near-isoelectric periods (suppressions). Prior work in neurophysiology suggests that burst and suppression segments are respectively associated with consumption and regeneration of adenosine triphosphate resource in cortical networks. This indicates that once a suppression (or, burst) segment begins, the propensity to switch out of the state gradually increases with duration spent in the state. Prior EEG monitoring frameworks that track the brain state during burst suppression by tracking the estimated fraction of time spent in suppression, relative to bursts, do not incorporate this information. In this work, we incorporate this information within a hidden semi-Markov model (HSMM) wherein two states (burst \& suppression) stochastically switch between each other using sojourn-time dependent transition probabilities. We demonstrate the HSMM's utility in analyzing clinical data by estimating the state probabilities, the optimal state sequence, and the brain's metabolic activation level characterized by parameters governing sojourn-time dependence in transition probabilities. The HSMM-based approach proposed here provides a novel statistical framework that advances the state-of-the-art in analyzing burst suppression EEG.
\end{abstract}

\section{INTRODUCTION}

Burst suppression is a characteristic feature of electroencephalogram (EEG) signals associated with states of profound unconsciousness in patients, such as those under general anesthesia or in coma [1]. This pattern comprises bursts of high amplitude electrical activity interspersed between isoelectric or quiescent suppression periods. Prior neurophysiological modeling studies indicate that the alternating burst and suppression epochs are respectively associated with adenosine triphosphate (ATP) depletion and regeneration [2]. In each cycle, the recovery of rhythms within bursts is associated with recovery of basal dynamics at the neuronal circuit level caused by transient increases in energy reserves. As the level of unconsciousness deepens, such as in general

\footnotetext{
*This work was partially supported by NIH Award P01-GM118629 and by funds from Massachusetts General Hospital (MGH) and the Picower Institute for Learning and Memory (PILM) (to ENB). SC is funded by the Picower Postdoctoral Fellowship.

${ }^{a}$ S. Chakravarty and T. Baum contributed equally to this work

${ }^{1}$ Picower Institute for Learning and Memory, Massachusetts Institute of Technology (MIT), Cambridge, MA 02139

${ }^{2}$ Departments of Electrical Engineering and Biology, the Pennsylvania State University

${ }^{3}$ Department of Brain and Cognitive Sciences, MIT

${ }^{4}$ Duke-NUS Graduate Medical School, Singapore

${ }^{5}$ Department of Anesthesia, Critical Care and Pain Medicine, MGH

${ }^{6}$ Institute of Medical Engineering and Science, MIT

${ }^{7}$ Harvard-MIT Division of Health Science and Technology
}

anesthesia, the brain's metabolic activation level characterizing the transient ATP dynamics progressively decreases. The fraction of time spent in the suppression periods within a local time window is regarded as an index of the level of brain's inactivation. The real time monitoring of this index in patients provides valuable information about a patient's anesthetic state during surgery or in medically-induced coma.

Early research efforts in quantitative description of burst suppression led to the burst suppression ratio (BSR) [3]. $\mathrm{BSR}$ is a value between 0 to 1 where $\mathrm{BSR}=0$ and $\mathrm{BSR}$ $=1$ respectively correspond to epochs with no suppressions and no bursts. Building upon the BSR, while addressing the limitations posed by the BSR-based analyses, an earlier work proposed the burst suppression probability (BSP) index which has better dynamical performance relative to BSR [4]. To estimate the BSP, the burst suppression EEG time series is viewed as a binary sequence which takes values of 1 when the EEG amplitude is below a user-specified threshold (data point belonging to a suppression segment) and 0 otherwise (data point belonging to a burst segment). This binary observation sequence allowed the authors to use a point process state-space (SS) model, where the binary observations were assumed to be realizations of a nonstationary binomial process, to estimate the BSP within a sequential Bayesian estimation framework [4].

The earlier BSP work did not incorporate mechanistic insight based on aforementioned ATP-dynamics from prior neurophysiological modeling work [2]. Utilizing this insight, a previous work posited a SS model with a nonlinear first order Markovian process describing the evolution of a continuous-valued latent state variable [5]. The binary observations were assumed to evolve as Markovian switchings between burst and suppression states where the transition probability depended on both the aforementioned latent state as well as the length spent in current burst or suppression state. Owing to the complexity induced by the nonlinear SS model, the model parameters were approximated by fitting a parametric distribution to the empirical distribution on the joint space of the smoothed BSP estimate and length of state epochs.

In the current work, we propose a novel, principled statistical modeling framework based on a hidden semi-Markov model (HSMM) [6], while preserving two key conceptual elements from the prior work [5], (1) the sojourn-time (time spent in the current state since entering that state) dependence of the transition probabilities governing the fast time-scale Markovian state switching and (2) the use of parameters that characterize this sojourn-time dependence as metrics 
to track the brain's metabolic activation level. One of the main advantages of our estimation paradigm over [5] is that the HSMM-based approach can estimate the metabolic activation level directly from the binary data without requiring the intermediate dependence on the BSP estimate, thus simplifying the estimation procedure. Another key advantage is that the HSMM formulation enables usage of standard sequential state estimation tools available in HMM literature [7] to track the unobserved burst/suppression states and their sojourn-times. It also provides an analytically tractable and computationally feasible framework to learn the sojourntime dependence of the state switching probabilities from data. We regard the parameters describing the sojourn-time dependence as indicative of the current metabolic activation level. Additionally, we use a Bayesian paradigm for HSMM model estimation to compensate for the finiteness of the data. We prescribe prior probability distributions on the model parameters and calculate the maximum aposteriori (MAP) estimates of these parameters using an Expectation Maximization (EM) framework.

\section{THEORY \\ A. Binary observation sequence}

We consider an EEG time-series data sampled at $F_{s} \mathrm{~Hz}$ that is converted to a binary time series by using an amplitude threshold such that any data point lying below the threshold is marked as 1 (suppression) and 0 (burst), otherwise. We view this binary sequence in non-overlapping time-windows of size $\Delta$ each containing $n\left(=F_{s} \Delta\right)$ binary observations. We denote the number of 1 's in the $k$-th time bin of size $\Delta$ as $y_{k}$ where $0 \leq y_{k} \leq n$. A sequence of length $K$, denoted by $y_{1: K} \triangleq\left\{y_{1}, y_{2}, \cdots, y_{K}\right\}$, is considered as the observation input to the HSMM model under consideration.

\section{B. HSMM observation model}

We assume that for the $k$-th time-bin of size $\Delta$, the observation $y_{k}$ is sampled from one of two binomial distributions.

$$
P\left(y_{k} \mid s_{k}=i\right)=\left(\begin{array}{c}
n \\
y_{k}
\end{array}\right)\left(p_{i}\right)^{y_{k}}\left(1-p_{i}\right)^{n-y_{k}}
$$

where, $i \in\{1,2\}$ and $s_{k}$ represents a discrete-valued random process such that $s_{k}=1$ (or, $s_{k}=2$ ) corresponds to a burst (or, suppression) state.

\section{HSMM state evolution model}

We assume that that the hidden (unobserved) random process $s_{k}$ is evolving stochastically, such that the probability to transition out of the current state depends on the timespent in the same state since entering that state. To characterize this, we define an integer-valued random processes $\tau_{k}$, such that a realization of $\tau_{k} \Delta$ is the sojourn-time for the realization $s_{k}$. We assume a finite sample space $\tau_{k} \in$ $\left\{1,2, \cdots, \tau_{\max }\right\}$ where $\tau_{\max }$ is set to a value that is high enough to include all possible sojourn-times in either state; a candidate value is $\tau_{\max }=K$. Using intuitions based on prior work [2], we assume that the propensity to switch out is lowest when a state switching occurs and gradually increases with time [2]. We model the sojourn-time dependence in the transition probabilities as follows,

$$
\begin{aligned}
& P\left(s_{k+1} \neq s_{k}, \tau_{k+1}=1 \mid s_{k}, \tau_{k}\right)=1-e^{-\lambda_{s_{k}}\left(\tau_{k}-1\right) \Delta} \\
& P\left(s_{k+1}=s_{k}, \tau_{k+1}=\min \left(\tau_{k}+1, \tau_{\max }\right) \mid s_{k}, \tau_{k}\right) \\
& =e^{-\lambda_{s_{k}}\left(\tau_{k}-1\right) \Delta}
\end{aligned}
$$

where the first and second equations correspond to switching and continuation events, respectively. For all the other transition events, beyond the ones accounted for in Eq. (2), the transition probabilities are always zero. Note that $\tau-1$ in the exponent ensures that when the process enters a given state, it tends to remain in that state for at least two $\Delta$ time-bins. We assume that over the time-window of size $\Delta_{B}=K \Delta$ the time-constant parameters $\lambda_{1}$ and $\lambda_{2}$ are constant.

\section{Likelihood}

We can write the joint distribution of $y_{1: K}$ as,

$$
P\left(y_{1: K}\right)=\sum_{s_{K}, \tau_{K}} P\left(s_{K}, \tau_{K}, y_{1: K}\right)
$$

and evaluate the likelihood using the following recursion,

$$
\begin{aligned}
P\left(s_{k}, \tau_{k}, y_{1: k}\right)= & P\left(y_{k} \mid s_{k}\right) \sum_{s_{k-1}, \tau_{k-1}} P\left(s_{k}, \tau_{k} \mid s_{k-1}, \tau_{k-1}\right) \\
& \cdot P\left(s_{k-1}, \tau_{k-1}, y_{1: k-1}\right)
\end{aligned}
$$

where, the observation and state transition probabilities are calculated per Eq. (1) and Eq. (2), respectively. The recursion in Eq. (4) begins with, $P\left(s_{1}, \tau_{1}, y_{1}\right)=P\left(y_{1} \mid s_{1}\right) \pi$, where $\pi \triangleq P\left(s_{1}, \tau_{1}\right)$, is the unconditional probability of the hidden states at $k=1$. Therefore, for the HSMM, the model parameter set includes $\Psi \triangleq\left\{\Theta, \Delta, \tau_{\max }\right\}$ where $\Theta \triangleq\left\{\pi, p_{1}, p_{2}, \lambda_{1}, \lambda_{2}\right\}$.

\section{E. Estimating states from observations}

Given a prescribed $\Psi$ we seek to estimate $P\left(s_{k}, \tau_{k} \mid y_{1: K}, \Theta\right)$ where the conditioning on $\Theta$ is indicated as it is estimated from data while the dependence on rest of the parameters in $\Psi$ is implied. We implement the HSMM as an extended state HMM [8] that enables us to use standard sequential Bayesian estimation procedure, Forward-Backward algorithm, to estimate the posterior state probabilities [7]. Using this same framework, we also calculate $P\left(s_{k}, \tau_{k}, s_{k+1}, \tau_{k+1} \mid y_{1: K}, \Theta\right)$ which is essential for the EM procedure. Furthermore, we use the Viterbi algorithm from HMM literature to estimate the MAP state trajectory [7],

$$
\left\{\hat{s}_{1: K}, \hat{\tau}_{1: K}\right\}=\operatorname{argmax} P\left(y_{1: K}, s_{1: K}, \tau_{1: K} \mid \Theta\right) .
$$

\section{F. MAP estimation of $\Theta$ from observations}

Given a prior probability, $P(\Theta)$, we determine pointestimates $\hat{\Theta}$ of $\Theta$ by maximizing the logarithm of the posterior probability,

$$
\hat{\Theta}=\operatorname{argmax} \ln P\left(\Theta \mid y_{1: K}\right)
$$


This optimization problem can be solved using the iterative EM procedure [9]. Taking a similar approach as [10], we maximize $R(\Theta, \hat{\Theta})$ defined as,

$$
R(\Theta, \hat{\Theta}) \triangleq Q(\Theta, \hat{\Theta})+\ln P(\Theta),
$$

in the M-step within an EM iteration where $\hat{\Theta}$ refers to the optimal estimate from the previous M-step. $Q(\Theta, \hat{\Theta})$ denotes the expected total data log-likelihood and defined as

$$
\begin{aligned}
Q(\Theta, \hat{\Theta}) \triangleq & \sum_{s_{1: K}, \tau_{1: K}} \ln P\left(y_{1: K}, s_{1: K}, \tau_{1: K} \mid \Theta\right) \\
& \cdot P\left(s_{1: K}, \tau_{1: K} \mid y_{1: K}, \hat{\Theta}\right)
\end{aligned}
$$

We assume statistically independent gamma priors on $\lambda_{1}$ and $\lambda_{2}$, beta priors on $p_{1}$ and $p_{2}$, and Dirichlet prior on $\pi$ as these are conjugate priors to exponential, binomial and multinomial distributions, respectively. These priors are represented as,

$$
\begin{aligned}
& P\left(\lambda_{i}\right) \propto \lambda_{i}^{a_{i}-1} e^{-b_{i} \lambda_{i}}, a_{i}>1, b_{i}>0, \\
& P\left(p_{i}\right) \propto\left(p_{i}\right)^{c_{i}-1}\left(1-p_{i}\right)^{d_{i}-1}, c_{i}, d_{i}>0, \\
& P(\pi) \propto \prod_{s=1}^{2} \prod_{\tau=1}^{\tau_{\max }}\left(\pi_{s, \tau}\right)^{e_{s, \tau}-1}, e_{s, \tau} \geq 0 .
\end{aligned}
$$

where, $i \in\{1,2\}$ and $\left\{a_{1}, b_{1}, a_{2}, b_{2}, c_{1}, d_{1}, c_{2}\right.$, $\left.d_{2},\left\{e_{s, \tau}\right\}_{s=1, \tau=1}^{2, \tau_{\max }}\right\}$ is the set of hyper-parameters that parameterize $P(\Theta)$. The combined prior is, $P(\Theta)=$ $P\left(\lambda_{1}\right) P\left(\lambda_{2}\right) P\left(p_{1}\right) P\left(p_{2}\right) P(\pi)$, which follows from the statistical independence assumption. Differentiating the expression $R(\Theta, \hat{\Theta})$ (Eq. (7)) with respect to each of the individual terms in $\Theta$ and equating the result to zero leads to the analytical representations of equations to solve for the updated $\hat{\Theta}$. While implementing each EM iteration, we perform the E-step to evaluate $P\left(s_{k}, \tau_{k} \mid y_{1: K}, \hat{\Theta}\right)$ and $P\left(s_{k}, \tau_{k}, s_{k+1}, \tau_{k+1} \mid y_{1: K}, \hat{\Theta}\right)$ using procedure discussed in Sec. II-E. Incorporating this information in the analytical expressions for the optimal $\Theta$, we solve for the new $\hat{\Theta}$ in the M-step.

\section{RESULTS}

We have analyzed our HSMM framework by applying it to de-identified EEG recordings from surgical patients under general anesthesia at Massachusetts General Hospital (MGH). The data was collected as a part of the MGH Human Research Committee-approved protocol. Each EEG dataset, sampled at a rate of $F_{s}=250 \mathrm{~Hz}$, is detrended over sliding windows of 2 seconds each, and then band-pass filtered from $1 \mathrm{~Hz}$ to $55 \mathrm{~Hz}$. After this preprocessing, each EEG data sequence is converted to a binary time series using an existing binary classification algorithm that is parameterized by a forgetting factor, $\beta$, and a classification threshold, $\theta$, on the instantaneous EEG variance [5]. We label time points with variance values lying below $\theta$ as 1 's and all others as 0 's. We use $\beta=.9534$ (the globally optimal forgetting factor in [5]) and $\theta=0.1 \mu V^{2}$, uniformly across all 3 patient datasets used here. We convert each of these binary sequences to a count sequence by summing the 1 's in nonoverlapping intervals, $\Delta=0.5 s \Rightarrow n=F_{s} \Delta=125$. In the subsequent analyses, unless otherwise prescribed, the default configuration for the MAP EM algorithm implementation will comprise $\Delta_{B}=30 \mathrm{~s} \Rightarrow K=60$ within which we have assumed stationarity of sojourn-time dependence in transition probabilities and $\tau_{\max }=60$.

For the beta priors on $p_{i}$ we choose $c_{1}=1.1, d_{1}=$ $20, c_{2}=20, d_{1}=1.1$ such that suppression (or, burst) segments will have a higher $P\left(y_{k} \mid s_{k}=2\right)$ (or, $P\left(y_{k} \mid s_{k}=\right.$ 1)) when $y_{k}$ 's are close to $n$ (or, 0 ) and lower when $y_{k}$ 's are close to 0 (or, $n$ ). The highly skewed beta priors ensure that states $s_{k}=1$ (and $s_{k}=2$ ) will correspond to bursts (and suppressions). For the gamma priors on $\lambda_{i}$, we specify $a_{1}=10=a_{2}$ and $b_{1}=90=b_{2}$ such that the distribution, $P\left(\lambda_{i}\right)$, has a mode $\left(=\left(a_{i}-1\right) / b_{i}\right)$ at $1 / 10$ and a wide spread around this mode. For the Dirichlet prior on $\pi$ we use $e_{s, \tau}=1 \forall s=\{1,2\}, \tau=\left\{1,2, \cdots, \tau_{\max }\right\}$. For the example cases analyzed here, an upper limit of $30 \mathrm{EM}$ iterations was found to be sufficient for $R(\Theta, \hat{\Theta})$ to converge.

To demonstrate the HSMM analyses' outputs on a single $\Delta_{B}$ segment, we analyze $0.5 \mathrm{~min}$ segments of a patient's EEG data. Comparing across the three segments in Fig. 1, we note that as the average duration of the suppression segments become shorter, the relative ratio $\hat{\lambda}_{2} / \hat{\lambda}_{1}$ tends to increase. This agrees with our intuition that shorter suppression segments in a given $\Delta_{B}$ will lead to higher $\lambda_{2}$ such that the switching probability approaches 1 more quickly.
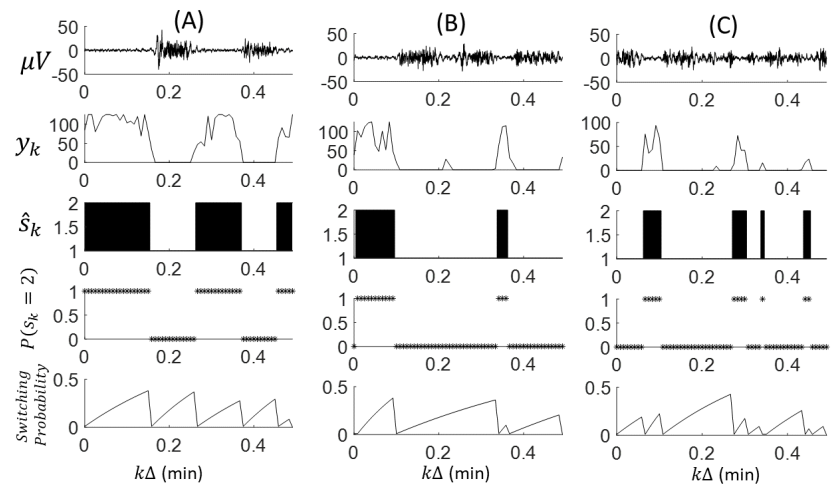

Fig. 1. HSMM estimated from three (A, B, C) 0.5 minute segments in a single recording session. Sub-panels, from top to bottom, represent the EEG data, observations $y_{k}$, the MAP estimate of state trajectory $\hat{s}_{1: K}$ (where the suppression segments are filled as black rectangles) given the converged $\hat{\Theta}, P\left(s_{k}=2 \mid y_{1: K}, \hat{\Theta}\right)$, and switching probability $\left(P\left(\hat{s}_{k+1} \neq\right.\right.$ $\left.\left.\hat{s}_{k} \mid \hat{s}_{1: K}, \hat{\Theta}\right)\right)$ given the MAP sequence, all as a function of time. The 4tuple $\left(\hat{\lambda}_{1}, \hat{\lambda}_{2}, \hat{p}_{1}, \hat{p}_{2}\right)$ for each case: A $(0.0751,0.0523,0.0208,0.7920)$, B (0.0313, 0.0940, 0.0321, 0.7331), C (0.0576, 0.1220, 0.0018, 0.3719).

To analyze the properties of the HSMM parameter estimator, we present a brief analyses in Fig. 2 based on data simulated using a ground truth HSMM similar to the one estimated in Fig. 1(A). Figs. 2(B) and 2(C) demonstrate that that our estimator for $\widehat{\lambda}$ and $\widehat{p}$ has desirable asymptotic properties; with increasing length of data, the posterior estimates become closer to the true value and the uncertainty reduces. This also agrees with our understanding that with more data, the posterior estimates will have relatively more contribution from the likelihood than the prior. To further explore the behavior of how $\lambda_{1}$ and $\lambda_{2}$ vary with duration 

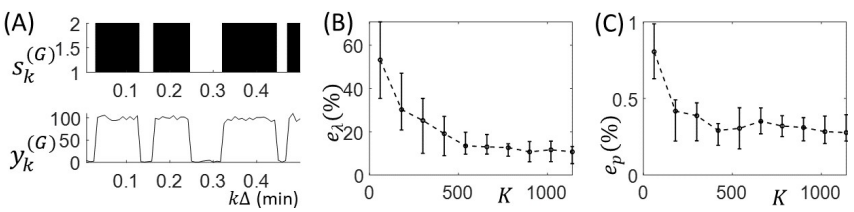

Fig. 2. Panel A - Random realization of length $K=60$ for an unobserved state $s_{k}^{(G)}$ and observation $y_{k}^{(G)}$ using a ground truth $\operatorname{HSMM}\left(\lambda^{(G)}=\right.$ $\left[\lambda_{1}^{(G)}, \lambda_{2}^{(G)}\right]=[0.07,0.05]$ and $\left.p^{(G)}=\left[p_{1}^{(G)}, p_{2}^{(G)}\right]=[0.02,0.80]\right)$. We generate such $y_{k}^{(G)}$ for 10 values of $K \in[60,1200]$, and for 20 replicates for each $K$. We run MAP HSMM estimation on each realization of $y_{k}^{(G)}$ (similar to rows 3 to 5 of Fig. 1), and then plot the median (black circles, joined by dashed line) and 25th and 75th percentiles of $e_{\lambda}$ (Panel B) and $e_{p}$ (Panel C) versus $K$ where, $e_{x}=100\left\|\hat{x}-x^{(G)}\right\|_{2} /\left\|x^{(G)}\right\|_{2}$.

of suppressions within fixed length $\Delta_{B}$ window, we apply HSMM to non-overlapping $\Delta_{B}$ windows on long recording sessions. In Fig. 3, by observing the MAP sequence of $\hat{s}_{1: K}$ we can see that time-points where the $y_{k}$ 's are close to zero (or, $n$ ) for prolonged time are classified as burst (or, suppression) segments. From each HSMM corresponding to every $\Delta_{B}$ window, we calculate the mean suppression probability as, $\bar{P}_{S} \triangleq \frac{1}{K} \sum_{k=1}^{K} P\left(s_{k}=2 \mid y_{1: K}, \hat{\Theta}\right)$. The $\bar{P}_{S}$ metric, like the BSR, is an estimate of the fraction of time spent in suppression state in the $\Delta_{B}$ bin, but more informed than BSR since it is based on conditional probability of suppression state given all data in $\Delta_{B}$ window and the MAP estimate of $\Theta$. Comparing across the three datasets, we note that wherever the black bars in the second row $\hat{s}_{1: K}$ grow denser we see increase in $\bar{P}_{S}$. The information on lengths of continuous suppression and burst segments is contained in $\hat{\lambda}_{1}$ and $\hat{\lambda}_{2}$ estimates. By visual examinations of $\hat{\lambda}$ timeseries, we note that for longer suppressions (or, bursts) the $\hat{\lambda}_{2}$ (or, $\hat{\lambda}_{1}$ ) is smaller, provided the analyses window has enough suppression events to move the MAP estimate of $\lambda_{2}$ away from the mode of the prior. This explains why we see $\hat{\lambda}_{2}$ stagnate at a high value when there are negligible or no suppression event in the epoch. To track the brain's metabolic activation state based on these time-constants, and inspired by [5], we define another metric, the relative duration index, $R D I \triangleq \hat{\lambda}_{2}^{-1} /\left(\hat{\lambda}_{1}^{-1}+\hat{\lambda}_{2}^{-1}\right)$, that encapsulates information of average duration of suppressions relative to bursts. The analyses presented in Fig. 3 indicates that on the slower timescale (across $\Delta_{B}$ time-windows) dynamics in $\bar{P}_{S}, R D I$ and $B S P$ may be similar.

\section{CONCLUSION}

In this work we have developed a novel, neurophysiologyinformed HSMM framework to model the stochastic switchings between EEG burst and suppression patterns. The estimated HSMM states can track the fast dynamics in state evolution $\left(s_{k}, \tau_{k}\right)$ evolving at $\Delta$ time-intervals. The HSMM model parameters $\left(\lambda_{1}, \lambda_{2}\right)$ can be used to track the changes in transition probability dependence on sojourn-time which is representative of dynamics in the brain's metabolic activation level. A key advantage of the MAP HSMM framework over previous works is that it provides an integrated inference framework for estimating state trajectories and their sojourntimes, as well as the sojourn-time dependence on state transitions. The latter aspect allows us to analyze duration statistics of the bursts and/or the suppressions across multiple $\Delta_{B}$ time-intervals. The sequential Bayesian inference
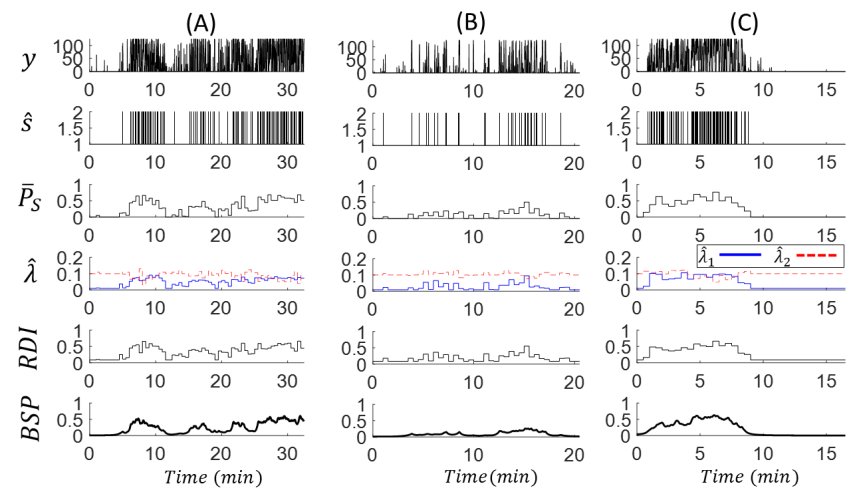

Fig. 3. HSMM analyses on 3 sessions of data (A, B, C). The rows, from top to bottom, indicate the observations $y_{k}$, the MAP estimate of state trajectory $\hat{s}_{1: K}$ (where the suppression segments are filled as black rectangles) given the converged $\hat{\Theta}$, the mean suppression probability $\bar{P}_{S}$, the MAP estimates of $\hat{\lambda}_{1}$ and $\hat{\lambda}_{2}$, the $R D I$, and $B S P$ estimate. The HSMM estimates are calculated from each of the sliding windows of size $\Delta_{B}$. Linear regression of $R D I$ (or, $B S P$ ) vs. $\bar{P}_{S}$ for each of three recordings A, B and C yields $R^{2}=0.90,0.85$ and 0.95 (or, $=0.88,0.66$ and 0.95 ), respectively.

framework of the HSMM makes it suitable for efficient real-time tracking of the aforementioned dynamical metrics. Although we have focused on a particular parametric form of sojourn-time dependence in transition probabilities (an exponential decay in continuation probability with sojourntime), our framework has scope for incorporating alternative descriptions and thus has potential as a statistical paradigm to compare across different models of sojourn-time dependence in transition probabilities.

\section{ACKNOWLEDGEMENT}

We thank anonymous reviewers for constructive feedback and M. B. Westover for sharing code for BSP estimation and pointing to relevant references.

\section{REFERENCES}

[1] E. N. Brown, R. Lydic, and N. D. Schiff, "General anesthesia, sleep, and coma," New England Journal of Medicine, vol. 363, no. 27, pp. 2638-2650, 2010, pMID: 21190458.

[2] S. Ching, P. L. Purdon, S. Vijayan, N. J. Kopell, and E. N. Brown, "A neurophysiological-metabolic model for burst suppression," Proceedings of the National Academy of Sciences, vol. 109, no. 8, pp. 3095-3100, 2012

[3] I. J. Rampil, R. B. Wlskopf, J. G. Brown, E. I. Eger, B. H. Johnson, M. A. Holmes, and J. H. Donegan, "I653 and Isoflurane Produce Similar Dose-related Changes in the Electroencephalogram of Pigs," Anesthesiology, vol. 69, pp. 298-302, 1988.

[4] J. Chemali, S. Ching, P. L. Purdon, K. Solt, and E. N. Brown, "Burst suppression probability algorithms: state-space methods for tracking EEG burst suppression,” Journal of Neural Engineering, vol. 10, no. 5, p. 056017, sep 2013.

[5] M. B. Westover, S. Ching, M. M. Shafi, S. S. Cash, and E. N. Brown, "Real-time segmentation and tracking of brain metabolic state in ICU EEG recordings of burst suppression," in Proc. of 35th Annual Intl. Conf. of the IEEE EMBC, July 2013, pp. 7108-7111.

[6] S. Z. Yu, "Hidden semi-Markov models," Artificial Intelligence, vol. 174, no. 2, pp. 215-243, 2010

[7] L. Rabiner, "A tutorial on hidden Markov models and selected applications in speech recognition," Proceedings of the IEEE, vol. 77, no. 2, pp. 257-286, 1989.

[8] V. Krishnamurthy, J. B. Moore, and S.-H. Chung, "On hidden fractal model signal processing," Signal Processing, vol. 24, no. 2, pp. 177 192, 1991.

[9] A. P. Dempster, N. M. Laird, and D. B. Rubin, "Maximum likelihood from incomplete data via the em algorithm," J. Royal Statistical Society. Series B (Methodological), vol. 39, no. 1, pp. 1-38, 1977.

[10] J.-L. Gauvain and C.-H. Lee, "Map estimation of continuous density hmm: Theory and applications," in Proceedings of the Workshop on Speech and Natural Language, ser. HLT '91. Stroudsburg, PA, USA: Association for Computational Linguistics, 1992, pp. 185-190. 